\title{
Efecto de la Gelatinización de Harina de Yuca sobre las Propiedades Mecánicas, Térmicas y Microestructurales de una Matriz Moldeada por Compresión
}

\author{
Elsa S. Cajiao, Luis E. Bustamante, Alcy R. Cerón y Héctor S. Villada \\ Universidad del Cauca, Facultad de Ciencias Agrarias, Vereda Las Guacas, Grupo de investigación \\ CYTBIA, Popayán - Colombia. (e-mail: alcyceron@unicauca.edu.co; hsamuel@yahoo.com)
}

Recibido Oct. 26, 2015; Aceptado Dic. 21, 2015; Versión final Feb. 20, 2016, Publicado Ago. 2016

\begin{abstract}
Resumen
En el presente estudio se evaluó el efecto del proceso de gelatinización de harina de yuca sobre las propiedades mecánicas, térmicas y microestructurales de una matriz semirrígida moldeada por compresión. Inicialmente se determinaron las condiciones que permitieron alcanzar el mayor grado de gelatinización de la harina en suspensiones acuosas calentadas controladamente. A partir de harina gelatinizada y sin gelatinizar, mezclada con fibra de fique, se elaboraron matrices moldeadas por compresión que fueron evaluadas en sus propiedades de flexión, absorción de agua, comportamiento térmico y microestructural. Las matrices elaboradas con harina gelatinizada presentaron mayores valores de esfuerzo y módulo de elasticidad en flexión, absorción de agua menor y tuvieron mejor estabilidad térmica. Adicionalmente, las superficies de las matrices fueron homogéneas y lisas. El proceso de gelatinización de la harina de yuca permitió una mayor termoplastificación e interacción entre los componentes de la matriz, lo que influyó sobre las propiedades evaluadas.
\end{abstract}

\section{Effect of Cassava Flour Gelatinization on the Mechanical, Thermal and Microstructural Properties of a Compression Molded Matrix}

\begin{abstract}
In the present study the effect of gelatinization process of cassava flour on the mechanical, thermal and microstructural properties of a semi-rigid compression molded matrix was evaluated. Initially the conditions that allowed the highest degree of gelatinization of the flour in controlled heated aqueous suspensions were determined. From ungelatinized and gelatinized flour mixed with fique fiber, molded matrices were prepared by compression. These were evaluated for their bending properties, water absorption, thermal and microstructural behavior. The matrices prepared with gelatinized flour had higher values of stress and flexural modulus, water absorption was smaller and had better thermal stability. Additionally, the surfaces of the matrices were homogeneous and smooth. The process of gelatinization of cassava flour allowed greater thermoplastic lamination and interaction between the components of the matrix, which influenced the evaluated properties.
\end{abstract}

Keywords: gelatinization; mechanical properties; thermal properties; semi-rigid matrix 


\section{INTRODUCCIÓN}

La harina de yuca es una alternativa importante para la fabricación de materiales amigables con el medio ambiente, por su alta disponibilidad, bajo costo y su alto contenido de almidón, el cual en condiciones de proceso específicas, presenta un comportamiento termoplástico (Navia et al., 2014). En efecto, los bioplásticos a base de almidón, se han convertido en una opción amigable para reemplazar los plásticos sintéticos derivados de la industria petroquímica, lo cual reduce la demanda de estos materiales, mitigando el impacto negativo sobre el medio ambiente. Sin embargo, el uso de almidón nativo dificulta el procesamiento de los plásticos biodegradables por lo que se hace necesario implementar procesos de modificación ya sea de tipo físico, químico o biotecnológico (Halley y Avérous, 2014).

La gelatinización es quizá el cambio físico de mayor importancia del almidón, consiste en una transición estructural de orden-desorden que sufren las cadenas poliméricas de este carbohidrato cuando es sometido a procesos de calentamiento en ambientes húmedos y acuosos, con gran impacto en el procesamiento, calidad y estabilidad de los productos basados en almidón (Acevedo et al., 2013). El proceso de gelatinización del almidón ocasiona una disminución en la energía de termoplastificación de matrices termoplásticas, generándose un descenso en la demanda energética y una reducción en los costos de operación (Tunjano et al., 2009). En productos elaborados con almidón, el proceso de gelatinización produce efectos tales como: redes más sólidas y estables térmicamente, distribución homogénea de la amilosa y amilopectina, mayor grado de cristalinidad y disminución en la permeabilidad al vapor de agua (Flores et al., 2007). La modificación física del almidón mediante gelatinización aumenta la termoplastificación de matrices obtenidas a partir de almidón, lo cual puede incrementar las propiedades mecánicas de los productos en cuanto a resistencia a la tensión, resistencia a la flexión, deformación y rigidez (Ramírez et al., 2011). Adicionalmente, la gelatinización permite la formación de redes con fuertes uniones intermoleculares, se reduce la tendencia a la retrogradación y se presenta una menor velocidad de absorción de agua (Pérez et al., 2007).

Estudios previos permitieron desarrollar una matriz moldeada a partir de harina nativa de yuca y fibra de fique, la cual fue valorada en cuanto a características mecánicas, térmicas y de absorción de humedad apropiadas para la elaboración de un empaque semirrígido biodegradable (Navia et al., 2015). El uso de fibras naturales aumentó las propiedades mecánicas de la matriz al actuar como un material de refuerzo debido a la fuerte unión que se desarrolla entre la interfaz de fibra-matriz por la similitud química del almidón de la harina con las fibras de celulosa (Ramírez et al., 2011; Navia et al., 2014). Sin embargo, se presentaron problemas en la matriz obtenida asociados con las pobres propiedades mecánicas y baja estabilidad a la humedad (Navia et al., 2014). Por lo tanto, el objetivo del presente trabajo fue evaluar el efecto de la gelatinización de harina de yuca sobre las propiedades físicas, mecánicas, térmicas y microestructurales de matrices termoplastificadas obtenidas mediante moldeo por compresión de mezclas de harina de yuca y fibra de fique.

\section{MATERIALES Y MÉTODOS}

Se describen los materiales y el procerso de gelatinización de la harina de yuca. Luego se explica la determinación del grado de gelatinización, yel análisis de las matrices moldeadas.

\section{Materiales}

Se utilizó harina de yuca variedad MBRA 383 con un contenido de almidón del 89,32\%, suministrada por DERIYUCA (Colombia), fibra de fique variedad Uña de Águila adquirida en la Cooperativa de Productores del municipio de Paniquitá (Cauca-Colombia) sometida a molienda y tamizaje hasta un tamaño comprendido entre $250 \mu \mathrm{m}$ y $450 \mu \mathrm{m}$. Como plastificante se utilizó glicerina grado comercial (pureza 99,7\%) suministrada por DISAN S.A. (Colombia).

\section{Gelatinización de la harina de yuca}

Se prepararon suspensiones a tres niveles distintos de concentración de harina de yuca en base seca (10, 15 y $20 \%$ ) y cada una se sometió a calentamiento durante 10 minutos a diversas temperaturas de estudio (67, 72 y $77^{\circ} \mathrm{C}$ ), manteniendo una velocidad de agitación constante de $16,75 \mathrm{rad} / \mathrm{s}$. El proceso de gelatinización se efectuó en una celda de empastamiento incorporada en un reómetro rotacional (TA Instruments, AR 1500ex) para garantizar un mejor control de los parámetros.

\section{Determinación del grado de gelatinización}

Se realizó la medición de la entalpia de gelatinización de cada una de las muestras de harina gelatinizada, en un calorímetro de barrido diferencial (TA Instruments, DSC Q20), aplicando un procedimiento de medición ajustado y adaptado de trabajos de otros investigadores (Cueto et al., 2011). Para cada medición 
se prepararon $25 \mathrm{mg}$ de mezcla a las concentraciones de sólidos de harina gelatinizada en agua destilada establecidas en la metodología anterior, se ubicaron en una capsula de aluminio hermética y se sometieron a calentamiento desde $20^{\circ} \mathrm{C}$ hasta $100{ }^{\circ} \mathrm{C}$ a una velocidad de calentamiento de $10^{\circ} \mathrm{C} / \mathrm{min}$. El grado de gelatinización se calculó comparando la entalpía de gelatinización medida para la muestra respecto a la entalpía de una muestra de harina sin gelatinizar, empleando la siguiente ecuación (Martínez et al., 2005):

Grado de gelatinización $(\%)=\left(1-\left(\frac{\Delta H g \text { muestra }}{\Delta H g \text { referencia }}\right)\right) * 100$

En esta ecuación, $\Delta H g$ muestra = Entalpia de gelatinización de la muestra $(\mathrm{J} / \mathrm{g})$ y $\Delta H g$ referencia $=$ Entalpia de gelatinización de la harina sin gelatinizar $(\mathrm{J} / \mathrm{g})$

\section{Obtención de matrices moldeadas de harina y fibra de fique}

Se gelatinizó harina de yuca calentando una suspensión de la harina en agua al 20\% (en base seca) a una temperatura de $67^{\circ} \mathrm{C}$, bajo agitación constante. Se preparó una mezcla incorporando $40 \mathrm{~g}$ de fibra de fique y $5 \mathrm{~g}$ de glicerol por cada $100 \mathrm{~g}$ de harina de yuca gelatinizada. La mezcla se revolvió durante 30 minutos en una máquina mezcladora (KitchenAid, USA) para homogeneizarla, después de lo cual se sometió a moldeo en una máquina de compresión hidráulica (EDAFA, Medellín, Colombia) a temperatura de $200{ }^{\circ} \mathrm{C}$ durante 3 minutos, bajo una presión de 6,89 MPa. Se obtuvieron muestras moldeadas (figura 1) que se sometieron a posterior análisis físico y mecánico. De forma similar se elaboraron muestras moldeadas de harina de yuca sin gelatinizar.

Fig. 1: Muestra de lámina moldeada obtenida a partir de harina de yuca gelatinizada y fique.

Análisis de las matrices moldeadas

Microscopía Electrónica de Barrido (SEM): Se registraron micrografías de la superficie de muestras de las matrices moldeadas, empleando un microscopio electrónico de barrido (SEM JSM-6490, USA) con filamento de tungsteno operado a $20 \mathrm{kV}$ con un nivel de magnificación de $50 \mathrm{X}$. Las muestras fueron previamente recubiertas con oro.

Resistencia a la flexión: Se evaluó la resistencia a la flexión de las matrices moldeadas obtenidas siguiendo la metodología definida por la norma ASTM D790-2010, empleando una máquina universal de ensayos (EZ$\mathrm{L}$, Shimadzu, Japan). Las matrices fueron moldeadas en forma de muestras planas de $127 \mathrm{~mm}$ de largo, $12,7 \mathrm{~mm}$ de ancho y de $2 \mathrm{~mm}$ de espesor. Previamente a la prueba, las muestras fueron acondicionadas durante 8 días a una humedad relativa de $50 \pm 10 \%$ y una temperatura de $23 \pm 2^{\circ} \mathrm{C}$.

Absorción de agua: Las mediciones se realizaron de acuerdo a la norma ASTM D570-1998. Se emplearon muestras moldeadas en forma de disco de $50,8 \mathrm{~mm}$ de diámetro y 3,2 $\mathrm{mm}$ de espesor. Las muestras se secaron a una temperatura de $50 \pm 3^{\circ} \mathrm{C}$ durante 24 horas, se registró su peso inicial en una balanza analítica (RADWAG XA 110/X, Polonia), se sumergieron en agua destilada a $23 \pm 1{ }^{\circ} \mathrm{C}$ durante 2 horas, se secó la superficie de las muestras y se registró nuevamente su peso. Debido a que las matrices elaboradas presentaron componentes solubles en agua, las muestras fueron secadas a $50 \pm 3{ }^{\circ} \mathrm{C}$ durante 24 horas y se pesaron nuevamente. El valor de absorción de agua se tomó como la suma del incremento del peso en la inmersión y del peso de la materia soluble en agua.

Análisis por calorimetría de barrido diferencial (DSC): Se cortó una porción de la matriz moldeada a analizar, entre $4 \mathrm{mg}$ a $5 \mathrm{mg}$, se encapsuló en cápsula de aluminio con cierre hermético y se introdujo al calorímetro de barrido diferencial (DSC Q20, TA Instruments, USA), en el cual la muestra se sometió a un rango de temperatura desde $-60^{\circ} \mathrm{C}$ hasta $250^{\circ} \mathrm{C}$ a una velocidad de $20^{\circ} \mathrm{C} / \mathrm{min}$. Se determinó la temperatura de fusión y entalpía de fusión de las matrices moldeadas. 


\section{Análisis termogravimétrico (TGA)}

De cada matriz se cortó una porción de muestra entre $4 \mathrm{mg}$ a $6 \mathrm{mg}$, se depositó en una cápsula de platino y se introdujo en el analizador termogravimétrico (TGA Q50, TA Instruments, USA). Las muestras fueron sometidas a calentamiento desde temperatura ambiente hasta $600{ }^{\circ} \mathrm{C}$ a una velocidad de $20{ }^{\circ} \mathrm{C} / \mathrm{min}$ en atmósfera inerte de nitrógeno para evaluar su estabilidad térmica.

\section{Análisis estadístico}

Se aplicaron análisis de varianza, seguidos por pruebas de Tukey, para evaluar las diferencias en los resultados del grado de gelatinización de la harina de yuca, verificada previamente la normalidad y la homogeneidad de las varianzas de los datos. Para establecer diferencias en los parámetros de resistencia a la flexión y absorción de agua de las matrices elaboradas, se aplicaron análisis mediante prueba t de Student, verificada previamente la normalidad de los datos. Se trabajaron tres replicas por experimento. Se utilizó un nivel de significancia $\alpha=0,05$. Los análisis fueron realizados en el paquete estadístico IBM SPPS Statistics 19.

\section{RESULTADOS Y DISCUSIÓN}

En la figura 2 se muestra el termograma comparado obtenido mediante calorimetría de barrido diferencial para muestras de harina gelatinizada $(\mathrm{HG})$ y sin gelatinizar (HSG). La entalpía, calculada como el área bajo la curva del pico endotérmico, representó la energía necesaria para llevar a cabo el proceso de gelatinización (Rodríguez et al., 2009; Liu et al., 2009). Según lo observado en la figura 2, la temperatura y la entalpia de gelatinización fueron menores para la muestra de harina gelatinizada, mientras que en la harina sin gelatinizar se presentó un valor de entalpía de gelatinización de 8,62 J/g cercano a valores de 8,3 y $10 \mathrm{~J} / \mathrm{g}$ reportados por otros investigadores (Martínez et al., 2005), probablemente por un mayor contenido de estructuras cristalinas en el almidón no gelatinizado, lo que exige una mayor cantidad de energía para gelatinizar sus gránulos. Estudios han demostrado que la pérdida de cristalinidad en almidones se genera cuando la gelatinización es completa (Chen et al., 2011; Torres et al., 2011; Carvalho, 2013), teniendo presente que esto depende del origen y naturaleza del almidón contenido en la harina (Torres et al., 2011). Por otro lado, los picos endotérmicos en los termogramas para muestras de almidones no gelatinizados oscilan en un intervalo de temperaturas entre 60 a $80^{\circ} \mathrm{C}$ (Chen et al., 2011), en el presente caso, para la muestra de harina sin gelatinizar este pico se dio a $67,1^{\circ} \mathrm{C}$.

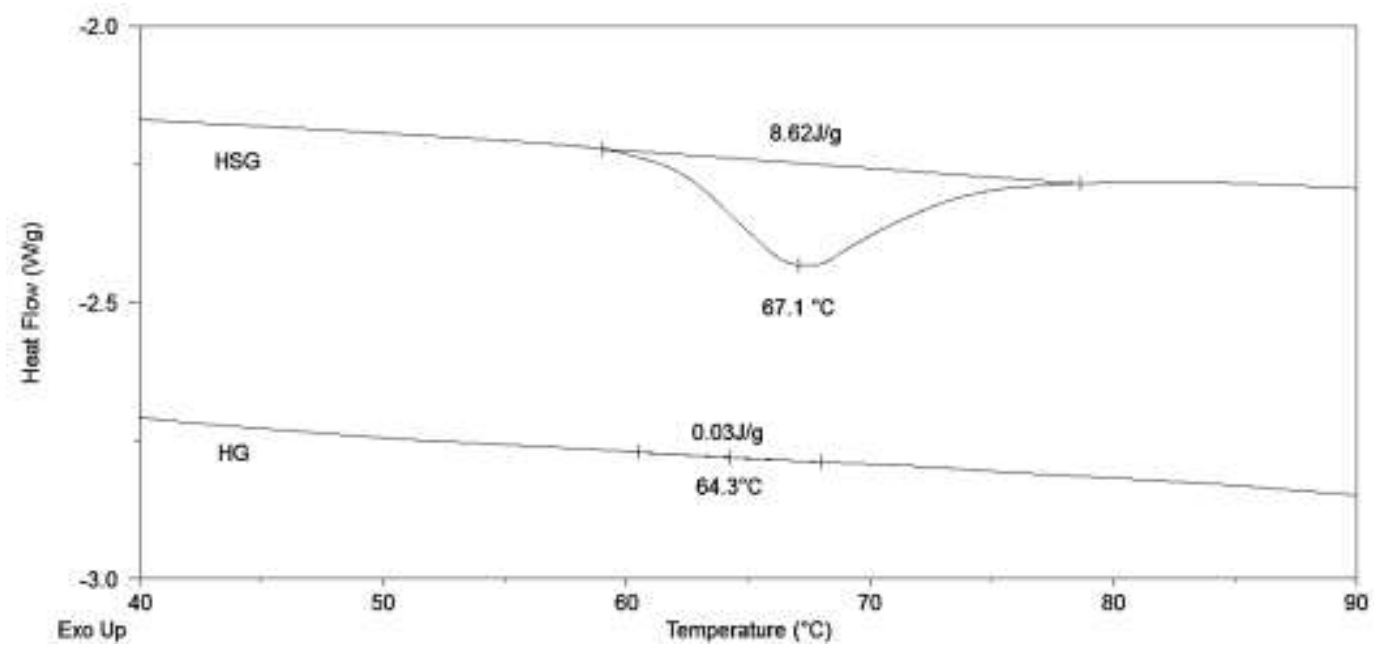

Fig. 2: Termograma de una muestra de harina gelatinizada (HG) y de harina sin gelatinizar (HSG).

Los valores obtenidos del grado de gelatinización calculado para cada una de las muestras se presentan en la tabla 1. El grado de gelatinización obtenido para las muestras evaluadas superó en general el valor de $99 \%$, lo que muestra que los procesos de gelatinización efectuados, utilizando los parámetros definidos para el estudio, fueron efectivos para lograr la gelatinización de la harina de yuca. Los tratamientos no presentaron diferencias significativas $(p<0,05)$ en el grado de gelatinización al cambiar la temperatura o la concentración de la harina en la suspensión según los parámetros establecidos en el proceso de gelatinización. Considerando lo anterior se decidió efectuar la gelatinización de harina de yuca con los respectivos componentes a $67{ }^{\circ} \mathrm{C}$ y $20 \%$ sólidos en suspensión, se escogió la menor temperatura de calentamiento para disminuir costos energéticos en el proceso y la mayor concentración de la suspensión 
que brindó una mayor consistencia en la harina obtenida para el posterior proceso de moldeo por compresión. En la Tabla 1 se reporta valor promedio \pm desviación estándar. Superíndices diferentes en la misma columna indican diferencias significativas $(p<0,05)$. HSG = harina sin gelatinizar.

Tabla 1: Grado de gelatinización según temperatura de calentamiento y concentración de la suspensión de HG.

\begin{tabular}{|c|c|c|c|}
\hline Tratamiento & $\begin{array}{c}\text { Temperatura de } \\
\text { calentamiento }\left({ }^{\circ} \mathrm{C}\right)\end{array}$ & $\begin{array}{c}\text { Concentración de la } \\
\text { suspensión }(\%)\end{array}$ & $\begin{array}{c}\text { Grado de } \\
\text { gelatinización (\%) }\end{array}$ \\
\hline 1 & 67 & 10 & $99,6 \pm 0,4^{\text {a }}$ \\
\hline 2 & 67 & 15 & $99,7 \pm 0,4^{\text {a }}$ \\
\hline 3 & 67 & 20 & $99,7 \pm 0,2^{\text {a }}$ \\
\hline 4 & 72 & 10 & $98,6 \pm 2,3^{\text {a }}$ \\
\hline 5 & 72 & 15 & $99,8 \pm 0,2^{\text {a }}$ \\
\hline 6 & 72 & 20 & $99,6 \pm 0,1^{\text {a }}$ \\
\hline 7 & 77 & 10 & $99,8 \pm 0,2^{\text {a }}$ \\
\hline 8 & 77 & 15 & $99,7 \pm 0,1^{\text {a }}$ \\
\hline 9 & 77 & 20 & $99,8 \pm 0,2^{\text {a }}$ \\
\hline HSG & - & - & 0,0 b \\
\hline
\end{tabular}

\section{Efecto de la harina gelatinizada}

En las zonas demarcadas de las micrografías de la figura 3 se puede observar que la matriz elaborada con harina gelatinizada (MHG) presenta una superficie más homogénea y lisa respecto a la matriz preparada con harina sin gelatinizar $(\mathrm{MH})$. En matrices elaboradas a partir de almidón mezclado con fibras naturales, se ha encontrado que la presencia de las fibras promueve la fusión de los gránulos del almidón, ocasionando una mayor termoplastificación de los productos finales (Versino et al., 2015) que puede ocasionar superficies más lisas en las matrices, tal como se obtuvo para la matriz con harina gelatinizada.

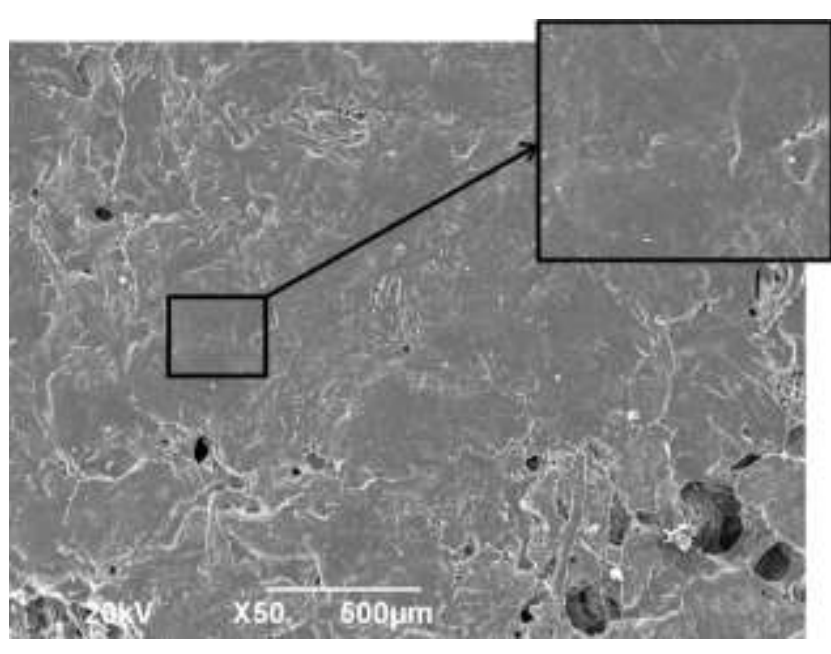

(a)

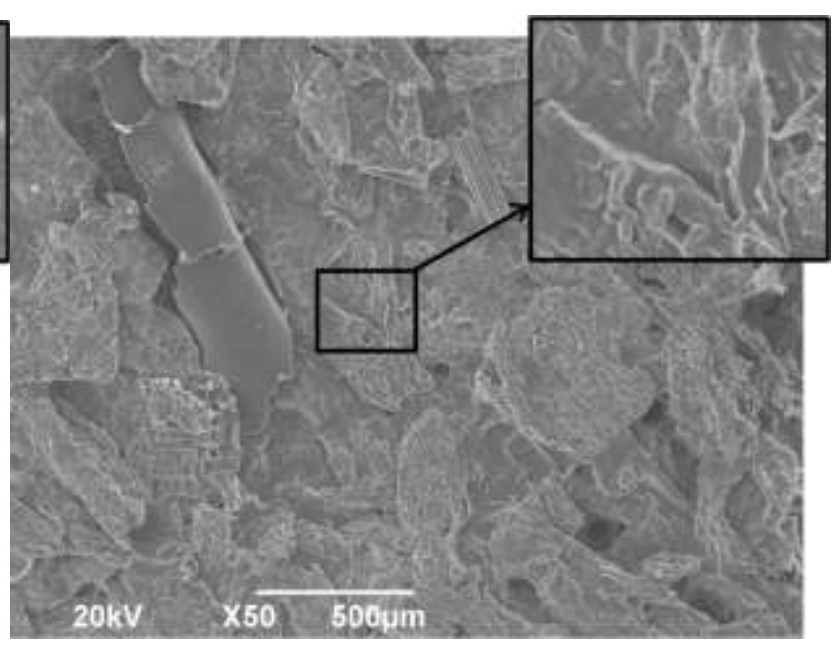

(b)

Fig. 3: Micrografías de las superficies de las matrices evaluadas. a) Matriz con harina gelatinizada; b) Matriz con harina sin gelatinizar.

En la matriz elaborada con harina gelatinizada hay una menor presencia de grietas y espacios intersticiales que denotan una mayor interrelación entre los componentes amiláceos y la fibra de fique, generándose un efecto de compactación entre la harina y la fibra de fique en la matriz (Ramírez et al., 2011; Ibrahim et al., 2014). Los grupos hidroxilo de la lignina, componente polimérico superficial de la fibra de fique, puede interactuar con el glicerol (plastificante) y con el almidón por medio de enlaces de hidrógeno fuertes que aumentan la compatibilidad interfacial de los componentes (López et al., 2015; Dias et al., 2011), relación que se ve favorecida en la harina gelatinizada en la cual las cadenas poliméricas del almidón están más libres y disponibles, debido al rompimiento de los gránulos de este polisacárido durante el proceso de gelatinización (Acevedo et al., 2013). 
Resistencia a la flexión. Los valores de esfuerzo máximo y módulo de elasticidad en flexión de la matriz moldeada a partir de harina de yuca gelatinizada (MHG) fueron superiores a los arrojados por la matriz de harina de yuca no gelatinizada $(\mathrm{MH})$, como se muestra en la tabla 2, ocasionado por una mejor plastificación de la estructura durante el proceso de moldeo por compresión, debido a una interacción más efectiva entre las fibras y las moléculas de amilosa y amilopectina liberadas durante la gelatinización, formando una matriz más compacta, uniforme y resistente respecto a la matriz (MH) (Ramírez et al., 2011; Paes et al., 2008; Ibrahim et al., 2014). En este sentido, el incremento de la interacción entre el almidón y la fibra de fique en la matriz moldeada (MHG) aumentó el efecto de refuerzo de la fibra (Ramírez et al., 2011; Ibrahim et al., 2014). En la Tabla 2 Se reporta valor promedio \pm desviación estándar. Superíndices diferentes en la misma columna indican diferencias significativas $(\mathrm{p}<0,05) . \mathrm{MHG}=$ matriz con harina gelatinizada. $\mathrm{MH}=\mathrm{matriz}$ con harina sin gelatinizar.

Según lo evidenciado por SEM, la superficie de la matriz con (MHG) (ver figura 3) fue más homogénea, lisa y con menos presencia de grietas, lo cual puede deberse a una fuerte interacción y mayor adhesión entre la harina gelatinizada y la fibra, debido a la similitud química entre el almidón de la harina y la celulosa de la fibra (Prachayawarakorn et al., 2013; Kaewtatip y Thongme, 2014), reflejando un efecto directo sobre las propiedades mecánicas en flexión. La presencia de grietas en matrices a base se almidones y fibras naturales son un factor adicional que origina pérdida en las propiedades mecánicas, evidenciándose una disminución en el módulo de elasticidad, posiblemente por causa de fallas internas (Navia et al., 2015; Prachayawarakorn et al., 2013; Ramírez et al., 2011).

Tabla 2: Propiedades mecánicas de flexión de las matrices moldeadas.

\begin{tabular}{|c|c|c|}
\hline Matriz & $\begin{array}{c}\text { Esfuerzo máximo en } \\
\text { flexión }(\mathrm{MPa})\end{array}$ & $\begin{array}{c}\text { Módulo de elasticidad en } \\
\text { flexión }(\mathrm{MPa})\end{array}$ \\
\hline $\mathrm{MHG}$ & $15,50 \pm 1,04^{\mathrm{a}}$ & $1712,27 \pm 295,16^{\mathrm{a}}$ \\
\hline $\mathrm{MH}$ & $3,06 \pm 0,24^{\mathrm{b}}$ & $245,06 \pm 24,37^{\mathrm{b}}$ \\
\hline
\end{tabular}

Absorción de agua. La absorción de agua y el aumento de volumen de los materiales compuestos a base de almidón es un fenómeno común en ambientes acuosos o húmedos, e influye en la integridad y desempeño de los productos terminados (Ramírez et al., 2011). En la tabla 3 se muestra cómo la matriz elaborada con harina gelatinizada presentó un valor significativamente menor de absorción de agua respecto a la matriz con harina sin gelatinizar. En la Tabla 3 se reporta valor promedio \pm desviación estándar. Superíndices diferentes en la misma columna indican diferencias significativas $(p<0,05)$. MHG = matriz con harina gelatinizada. $\mathrm{MH}=$ matriz con harina sin gelatinizar. La mayor plastificación de la harina gelatinizada disminuyó los espacios libres y la porosidad en la matriz, reduciendo la absorción de agua por una menor presencia de espacios internos debidos a la gelatinización (Müller et al., 2014). Además la interacción entre el almidón gelatinizado con la fibra, disminuyó la absorción de agua al aumentarse el efecto hidrofóbico sobre la matriz de la celulosa presente en las fibras (Ibrahim et al., 2014).

Tabla 3: Porcentaje de absorción de agua de las matrices moldeadas.

\begin{tabular}{|c|c|}
\hline Matriz & Absorción de agua (\%) \\
\hline MHG & $173,22 \pm 6,04^{\mathrm{a}}$ \\
\hline $\mathrm{MH}$ & $250,67 \pm 17,78^{\mathrm{b}}$ \\
\hline
\end{tabular}

\section{Análisis térmico por calorimetría de barrido diferencial}

En la figura 4 se muestra un termograma en el cual se comparan las señales de fusión observadas para las dos matrices evaluadas y en la tabla 4 se reportan los valores promedio de temperatura y entalpía de fusión para las señales encontradas. En la figura 4 se puede observar que para ambas matrices se presentaron dos señales de fusión. La aparición de múltiples señales endotérmicas es común en almidones con baja humedad, tal como ocurre en las matrices cuya humedad final es menor al 4\%, debido a la distribución heterogénea del agua dentro de la matriz o a la generación de dominios cristalinos que presentan diferente estabilidad térmica (Bertolini, 2010). En la Tabla 4 se reporta valor promedio \pm desviación estándar. $\mathrm{MHG}=$ matriz con harina gelatinizada. $\mathrm{MH}=$ matriz con harina sin gelatinizar.

Comparando los valores de las temperaturas de fusión de las señales para las dos matrices, se observa que existe un incremento en la temperatura para la señal 2 en la matriz MHG. Este aumento en la temperatura de fusión en las muestras preparadas con harina de yuca gelatinizada se debe a un incremento en la estabilidad de las zonas cristalinas formadas en la matriz termoplástica, debido a una mayor formación de enlaces hidrógeno entre el almidón gelatinizado y los demás componentes de la matriz (Luo et al., 2012). Además la gelatinización permite el rompimiento de estructuras cristalinas nativas del gránulo de almidón lo cual deja libre 
grupos hidroxilo que pueden interactuar con otros componentes de la matriz o con otras cadenas poliméricas diferentes (Chen et al., 2011; Qiao et al., 2011). También se puede observar que el pico de fusión de la matriz HG es más angosto respecto a las muestras de HSG, posiblemente por la formación de estructuras cristalinas más organizadas y uniformes al interior de la matriz polimérica (López et al., 2014).

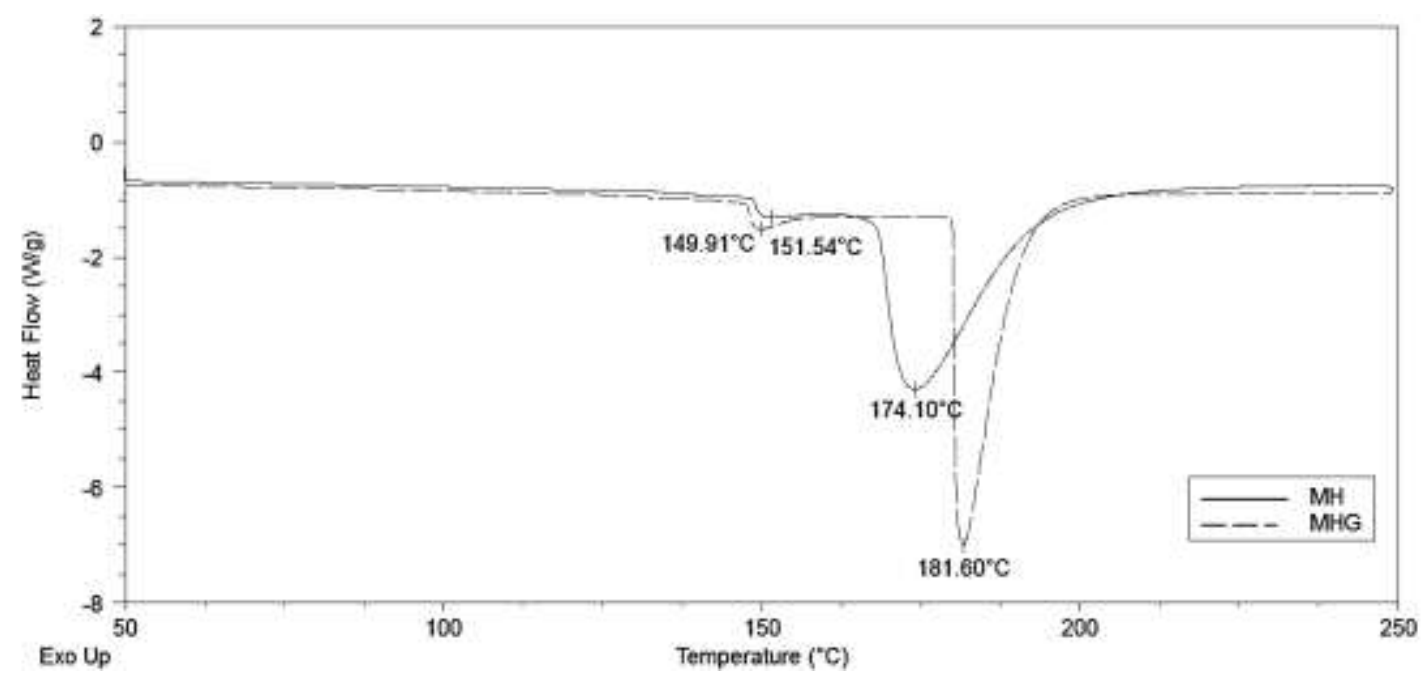

Fig. 4: Termograma de las matrices moldeadas con harina gelatinizada ( $\mathrm{MHG})$ y con harina sin gelatinizar $(\mathrm{MH})$.

Tabla 4: Temperaturas y entalpías de fusión de las matrices.

\begin{tabular}{|c|c|c|c|c|}
\hline Matriz & $\begin{array}{c}\text { Temperatura de } \\
\text { fusión señal 1 }\left({ }^{\circ} \mathrm{C}\right)\end{array}$ & $\begin{array}{c}\text { Temperatura de } \\
\text { fusión señal 2 }\left({ }^{\circ} \mathrm{C}\right)\end{array}$ & $\begin{array}{c}\text { Entalpia de fusión } \\
\text { señal 1 }(\mathrm{J} / \mathrm{g})\end{array}$ & $\begin{array}{c}\text { Entalpia de } \\
\text { fusión señal 2 } \\
(\mathrm{J} / \mathrm{g})\end{array}$ \\
\hline $\mathrm{MHG}$ & $150,24 \pm 0,46$ & $185,14 \pm 5,00$ & $13,76 \pm 0,16$ & $110,25 \pm 10,68$ \\
\hline $\mathrm{MH}$ & $153,25 \pm 2,41$ & $176,84 \pm 3,87$ & $7,52 \pm 2,45$ & $132,35 \pm 21,99$ \\
\hline
\end{tabular}

\section{Estabilidad térmica de las matrices}

En las figuras 5 y 6 se muestran ejemplos de termogramas obtenidos mediante TGA para las dos matrices evaluadas, en esta figuras se observa que en las curvas de cambio de peso respecto al cambio de temperatura, se presentan perdidas de peso en la zona de alta volatilidad ( $\mathrm{T}$ ambiente hasta $150^{\circ} \mathrm{C}$ ) y en la zona de media volatilidad $\left(150^{\circ} \mathrm{C}\right.$ hasta $\left.600^{\circ} \mathrm{C}\right)$.

En este tipo de matrices las sustancias que primero se liberan al calentar las muestras son agua, plastificantes y fragmentos de almidón degradado térmicamente en el proceso de moldeo por compresión, que hacen parte de los componentes de alta volatilidad (Luo et al., 2012). Comparando los contenidos de componentes de alta volatilidad entre las matrices (tabla 5), se observa que la matriz preparada con harina sin gelatinizar tiene una mayor cantidad de estos, lo que puede ser provocado por la incorporación de agua del ambiente en el interior de esta matriz considerando que ella tiene una mayor capacidad de absorción de agua, como también puede ser debido a la mayor presencia de residuos de almidón producidos por degradación térmica que sufre esta matriz al requerir mayor energía para su plastificación (Versino et al., 2015). En lo que respecta a los contenidos de los componentes de media volatilidad estos fueron similares entre las matrices. En la zona de media volatilidad ocurre la degradación del almidón (Luo et al., 2012) y de las estructuras celulósicas de la fibra de fique (Ramírez et al., 2011; Pfister y Larock, 2010). En la Tabla 5 Se reporta valor promedio \pm desviación estándar. $\mathrm{MHG}=$ matriz con harina gelatinizada. $\mathrm{MH}=\mathrm{matriz}$ con harina sin gelatinizar.

Tabla 5: Contenido y temperaturas de máxima degradación para los componentes determinados por TGA para las matrices

\begin{tabular}{|l|c|c|c|c|c|c|}
\hline \multirow{3}{*}{ Matriz } & \multicolumn{2}{|c|}{$\begin{array}{c}\text { Componentes de alta } \\
\text { volatilidad }\end{array}$} & \multicolumn{3}{|c|}{ Componentes de media volatilidad } & Residuo \\
\cline { 2 - 7 } & $\begin{array}{c}\text { Contenido } \\
(\%)\end{array}$ & $\begin{array}{c}\text { Temperatura } \\
\text { de máxima } \\
\text { degradación } \\
\left({ }^{\circ} \mathrm{C}\right)\end{array}$ & $\begin{array}{c}\text { Contenido } \\
(\%)\end{array}$ & $\begin{array}{c}\text { Temperatura 1 } \\
\text { de máxima } \\
\text { degradación } \\
\left({ }^{\circ} \mathrm{C}\right)\end{array}$ & $\begin{array}{c}\text { Temperatura } 2 \\
\text { de máxima } \\
\text { degradación } \\
\left({ }^{\circ} \mathrm{C}\right)\end{array}$ & Contenido (\%) \\
\hline $\mathrm{MH}$ & $10,53 \pm 0,05$ & $54,45 \pm 12,55$ & $78,27 \pm 2,83$ & $310,60 \pm 0,99$ & $355,90 \pm 1,87$ & $11,20 \pm 2,79$ \\
\hline $\mathrm{MHG}$ & $9,46 \pm 0,46$ & $50,33 \pm 4,06$ & $80,89 \pm 1,11$ & $313,59 \pm 0,70$ & $364,51 \pm 0,67$ & $9,64 \pm 1,04$ \\
\hline
\end{tabular}




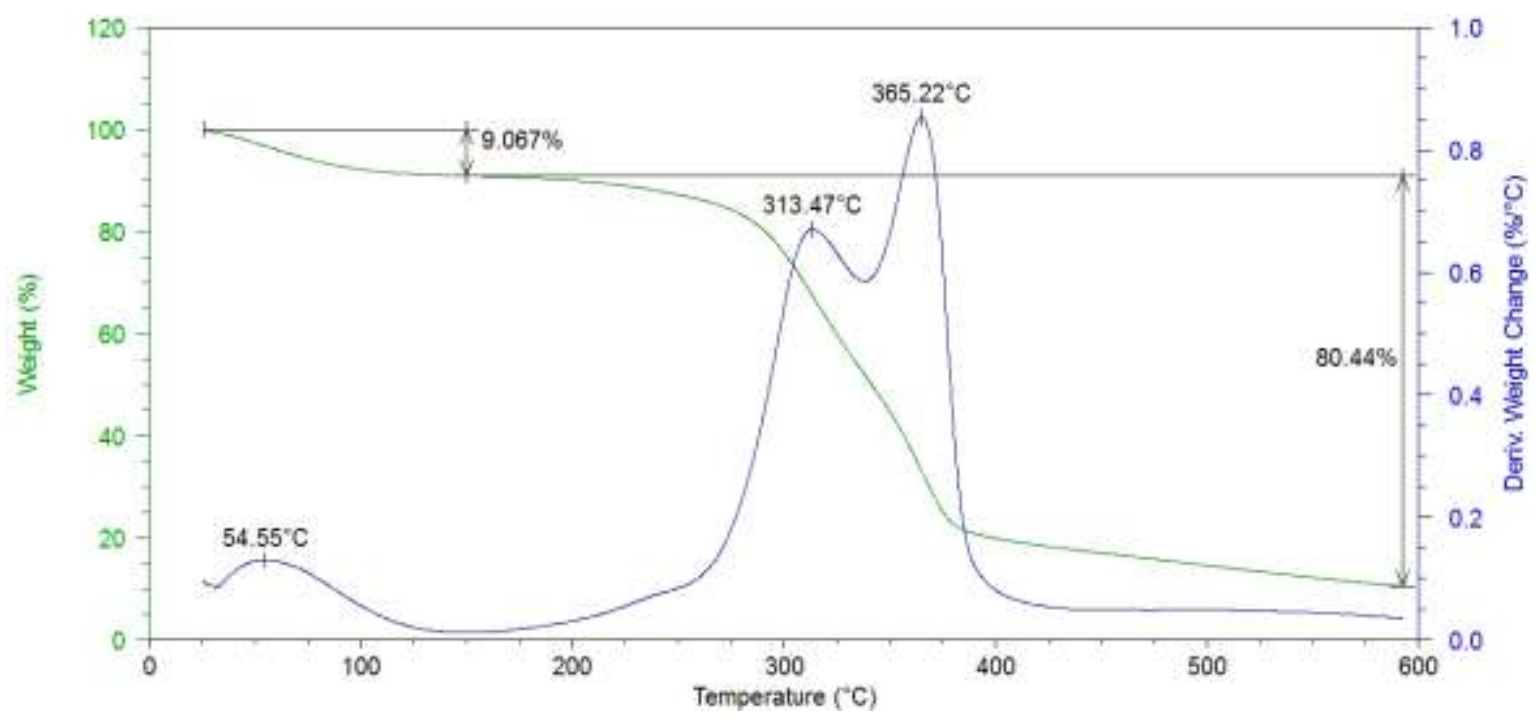

Fig.5: Termograma obtenido por TGA para la matriz MHG.

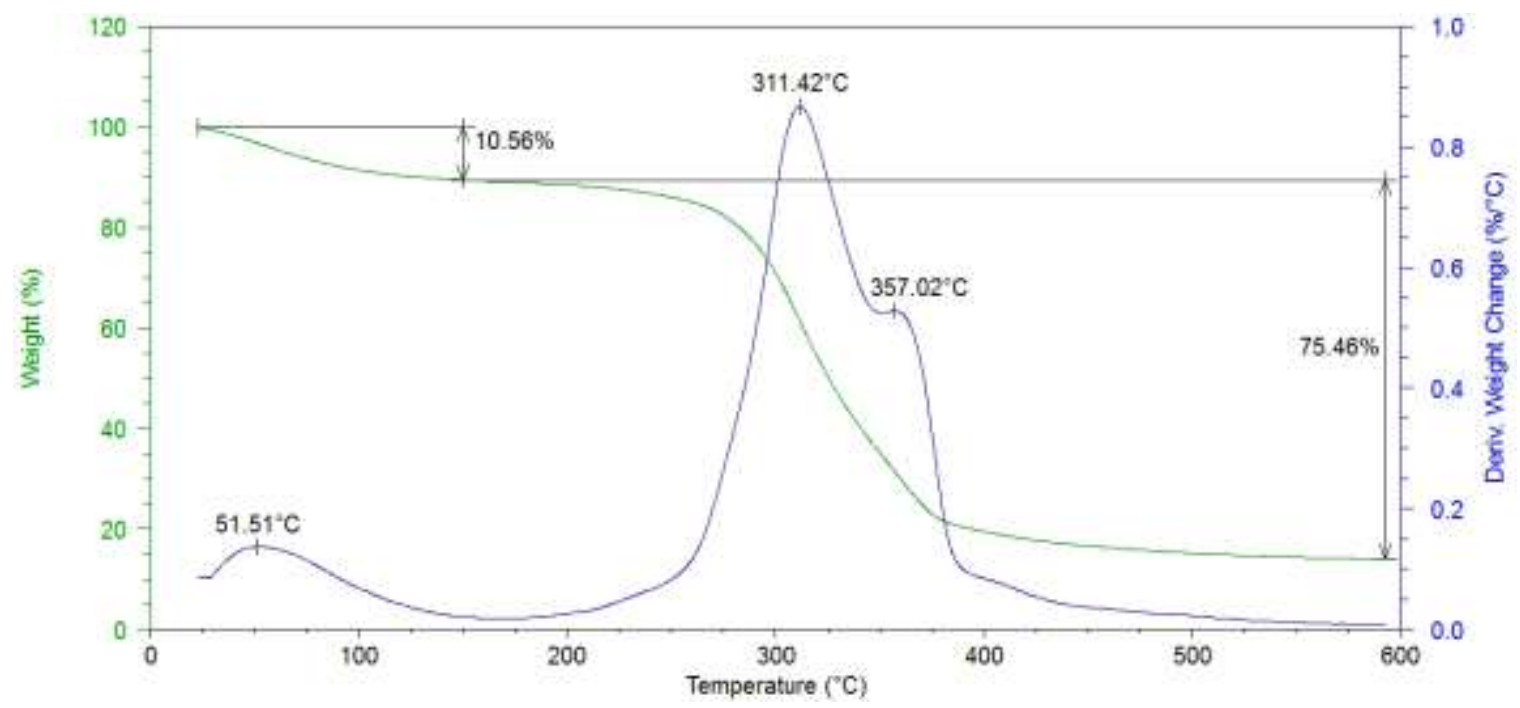

Fig. 6: Termograma obtenido por TGA para la matriz $\mathrm{MH}$.

Al representar la primera derivada del cambio del peso respecto a la temperatura (figuras 5 y 6 ), se encuentra que para ambas matrices se presenta un pico de máxima degradación en la zona de alta volatilidad y dos picos de degradación en la zona de media volatilidad.

En lo referente a la zona de media volatilidad, se tiene que el pico o señal de degradación a más baja temperatura corresponde a la descomposición del almidón presente en la harina de yuca, lo que se comprobó efectuando el análisis termogravimétrico a la harina de yuca sola que dio un valor de $305,41^{\circ} \mathrm{C}$. La señal con la mayor temperatura está relacionada con la descomposición de la fibra de fique, evidenciado en el análisis de la fibra sola $\left(\mathrm{T}=369,94^{\circ} \mathrm{C}\right)$. Un primer hecho relevante que se nota en los termogramas (figuras 5 y 6), es que la altura de la señal del pico vinculado con la degradación del almidón es menor para la matriz con harina gelatinizada respecto a la matriz con harina sin gelatinizar, comportamiento que se da a la par con el incremento en la altura del pico de degradación de la fibra de fique para la matriz con harina gelatinizada (MHG). Este cambio en la alturas de las señales de degradación es provocado por un aumento en la estabilidad térmica del almidón dado por una mayor interacción entre este componente con la fibra (Cheng et al., 2015), esto favorecido por la gelatinización de la harina que hace que el almidón tienda a degradarse en conjunto con la fibra de fique a una temperatura mayor.

En general, los valores de las temperaturas de las señales de degradación en la zona de media volatilidad (tabla 5) son mayores para la matriz elaborada con harina gelatinizada. Tal como ha mencionado previamente, el aumento de la interacción entre el almidón y la fibra de fique, promovido por el tratamiento previo de gelatinización de la harina, ocasiona un aumento general de estabilidad térmica de la matriz con harina gelatinizada (Kaewtatip y Thongmee, 2014; Narkchamnan y Sakdaronnaron, 2013). 


\section{CONCLUSIONES}

A partir de los resultados obtenidos se concluye lo siguiente: 1) La matriz elaborada con harina gelatinizada generó mayores propiedades mecánicas de flexión respecto a la matriz con harina no gelatinizada, dada la mayor termoplastificación del producto debido a la gelatinización, lo cual permitió obtener matrices más homogéneas en su superficie y con menor porosidad, observado por microscopía electrónica de barrido. 2) El proceso de gelatinización de la harina de yuca influyó significativamente en la absorción de agua de la matriz moldeada a base de harina de yuca, la tendencia fue hacia un menor valor de esta propiedad en la matriz fabricada con harina de yuca gelatinizada. 3) El tratamiento de gelatinización de la harina promovió la interacción entre los componentes de las matrices elaboradas, se aumentó la temperatura de fusión de las muestras e igualmente se incrementó la temperatura de degradación térmica del conjunto harina de yuca y fibra de fique. En general, se observó que el proceso de gelatinización de la harina de yuca permitió una mayor termoplastificación e interacción entre los componentes de la matriz semirrígida, lo que influyó positivamente sobre las propiedades evaluadas, lo que brinda la posibilidad que esta matriz pueda ser utilizada en la fabricación de empaques y embalajes, para lo cual se requieren estudios adicionales.

\section{AGRADECIMIENTOS}

Los autores agradecen el apoyo del departamento administrativo de Ciencia, Tecnología e Innovación COLCIENCIAS, el Sistema General de Regalías y la Universidad del Cauca.

\section{REFERENCIAS}

Acevedo, E. y otros tres autores, Características del almidón de maíz y relación con las enzimas de su biosíntesis, Agrociencia, 47(1), 1-12 (2013)

Bertolini, A.C., Starches. Characterization, Properties and Applications, CRC Press, 75-78, New York, USA (2010)

Carvalho, J.F., Starch major sources, Properties and applications as thermoplastic materials, Handbook of biopolymers and biodegradable plastics, 129-152 (2013)

Chen, P. y otros cinco autores, Internal structures and phase-transitions of starch granules during gelatinization, Carbohydrate Polymers, 83, 1975-1983 (2011)

Cheng, W. y otros seis autores. Research on thermoplastic starch and diferente fiber reinforced biomass composites, RSC Advances, 5, 49824-49830 (2015)

Cueto, D. y otros tres autores, Efecto de la adición de harina de yuca (Manihot esculenta Crantz) sobre las características sensoriales, reológicas y físicas de tortas y panquecas, Revista de la Facultad de Agronomía, 3 (2), 64-74 (2011)

Dias, A.B. y otros tres autores, Mechanical and barrier properties of composite films based on rice flour and cellulose fibers, LWT - Food Science and Technology, 44(2), 535-542 (2011)

Flores, S. y otros cuatro autores, Physical properties of tapioca-starch edible films: Influence of filmmaking and potassium sorbate, Food Research International, 40 (2), 257-265 (2007)

Halley P.J. y L.R. Avérous., Starch Modification, Starch polymers: from genetic engineering to green applications, 1 era edición, Elsevier, 29-30,San Diego, USA (2014)

Ibrahim, H. y otros tres autores, Characteristics of starch-based biodegradable composites reinforced with date palm and flax fibers, Carbohydrate Polymers, 101, 11-19 (2014)

Kaewtatip, K. y J. Thongmee, Preparation of thermoplastic starch/treated bagasse fiber composites, StarchStärke, 66 (7-8), 724-728 (2014)

López, M.A. y otros cuatro autores, Thermo-compression of biodegradable thermoplastic corn starch films containing chitin and chitosan, LWT - Food Science and Technology, 57, 106-115 (2014)

López, O.V. y otros tres autores, Agro-industrial residue from starch extraction of Pachyrhizus ahipa as filler of thermoplastic corn starch films, Carbohydrate Polymers, 134, 324-332 (2015)

Liu, H. y otros cinco autores, Thermal processing of starch-based polymers, Progress in Polymer Science, $34,1348-1368$ (2009) 
Luo, X., J. Li. y X. Lin, Effect of gelatinization and additives on morphology and thermal behavior of corn starch/PVA blend films, Carbohydrate Polymers, 90, 1595-1600 (2012)

Martínez, F. y otros tres autores, Preparación y propiedades de almidones pregelatinizados de yuca (Manihot esculenta crantz) y jícama (pachyrhizus erosus) usando calentamiento óhmico, Agrociencia, 39, 275-283 (2005)

Müller, P. y otros cuatro autores, El almidón termoplástico compuestos de madera: Interacciones interfaciales y propiedades funcionales, Polímeros de Hidratos De carbono, 102, 821-829 (2014)

Narkchamnan, S. y C. Sakdaronnaron, Thermo-molded biocomposite from cassava starch, natural fibers and lignin associated by laccase-mediator system, Carbohydrate Polymers, 96, 109 - 117 (2013)

Navia, D.P., A.A. Ayala. y H.S. Villada, Adsorción de Vapor de Agua de Bioplásticos Elaborados con Harina de dos Variedades de Yuca (Adsorción Manihot esculenta Crantz), Información tecnológica, 25 (6), 23-32 (2014)

Navia, D.P., A.A. Ayala. y H.S. Villada, Biocompuestos de Harina de Yuca obtenidos por termocompresión. Efecto de las Condiciones de Proceso, Información Tecnológica, 26 (5), 55-62 (2015)

Paes, S.S., I. Yakimets. y J.R. Mitchell, Influence of gelatinization process on functional properties of cassava starch films, Food Hydrocolloids, 22, 788-797 (2008)

Pérez, E. y otros tres autores, Production and characterization of Cassava (Manihot esculenta crantz) flour using different thermal treatments, Interciencia, 32(9), 615-619 (2007)

Pfister, D. y R. Larock, Thermophysical properties of conjugated soybean oil/corn stover biocomposites, Bioresorce Technol, 101(15), 6200-6206 (2010)

Prachayawarakorn, J. y otros tres autores, Effect of jute and kapok fibers on properties of thermoplastic cassava starch composites, Materials and Design, 47, 309-315 (2013)

Qiao, X.Y., Z.Z. Tang. y K. Sun, Plasticization of corn starch by polyol mixtures, Carbohydrate Polymers, 83, 659-664 (2011)

Ramírez, M.G. y otros cinco autores, Study of the properties of biocomposites. Part I. Cassava starch-green coir fibers from Brazil, Carbohydrate Polymers, 86, 1712-1722 (2011)

Rodríguez, P., E. San Martín. y G. González de la Cruz, Calorimetría diferencial de barrido y rayos-x del almidón obtenido por nixtamalización fraccionada, Sociedad Mexicana de Ciencia de Superficies y de Vacío, 13, 61-65 (2009)

Torres, F.G. y otros tres autores, Morphological and thermal characterization of native starches from Andean crops, Starch/Starke, 63, 381-389 (2011)

Tunjano, V. y otros cinco autores, Estudio de las propiedades térmicas y mecánicas del almidón termoplástico (TPS) reforzado con nanoarcilla, Suplemento de la Revista Latinoamericana de Metalurgia y Materiales, 1, 29-36 (2009)

Versino, F., O.V. López. y M.A. García, Sustainable use of cassava (Manihot esculenta) roots as raw material for biocomposites development, Industrial Crops and Products, 65, 79-89 (2015) 